\title{
Quantification of the articular view of the elbow afforded by standard portals using $30^{\circ}$ arthroscopy
}

\author{
Robert Moverley', Henry B. Colaco', Bran Rudran', \\ Matthew Szarko ${ }^{2}$, Magnus Arnander ${ }^{3}$ and Duncan Tennent ${ }^{3}$
}

\begin{abstract}
Background: The present study quantifies the field of view (FOV) from standard arthroscopy portals and aims to identify anatomical regions where the FOV is limited.

Methods: Eleven cadaveric elbows were examined through standard anteromedial, anterolateral and posterior portals. The FOV was marked with dye using a spinal needle. The articular surfaces were then exposed and the percentage FOV seen was calculated. FOV percentage areas were compared using a Student's $t$-test (JMP, version 10, SAS Institute Inc., Cary, NC, USA). $P<0.05$ was considered statistically significant.

Results: The mean (SD) FOV percentage area seen from the anteromedial portal and anterolateral portal was $91.69 \%$ (3.63\%) and $92.03 \%$ (3.93\%), respectively, for the anterior humerus articular surface. There was no significant difference in the mean FOV percentage area seen from the anteromedial and anterolateral portals $(p=0.99)$. The mean (SD) FOV percentage area of the posterior humerus articular surface was $84.69 \%(2.28 \%)$. The mean (SD) FOV percentage area seen of the radial head and trochlear was $16.05 \%(2.66 \%)$ and $4.14 \%$ (I.76\%), respectively.

Conclusions: The present study is the first to quantify the FOV of elbow arthroscopy. The majority of the anterior and posterior humerus articular surface can be seen through standard portals. The limitations in FOV are primarily confined to the radial head and trochlear notch.
\end{abstract}

Keywords

$30^{\circ}$ arthroscope, elbow arthroscopy, field of view

Date received: Ist June 2016; accepted: 29th November 2016

\section{Introduction}

Subsequent to being described by Andrews and Carson $^{1}$ in 1985 , the indications for elbow arthroscopy have significantly expanded to include capsular release, tennis elbow release and fracture fixation. Despite this, it is recognized that the procedures are technically difficult and that the associated risks of injury to periarticular structures are not insignificant.

Several studies have suggested that some regions of the elbow are unseen or difficult to reach during elbow arthroscopy. ${ }^{1-3}$ Despite it being well recognized that the afforded view is often limited, there has been little published research into quantifying the limits of the field of view (FOV) in elbow arthroscopy.
A number of portals have been described in the literature (Table 1), ${ }^{4-6}$ although these have been developed primarily to minimize the risk of damage to the adjacent neurovascular structures ${ }^{7}$ as opposed to maximizing viewing potential.

The current literature regarding the consequences of the limitation on FOV is generally qualitative and descriptive. The original paper by Andrews and

\footnotetext{
'Trauma \& Orthopaedic Surgery, St George's Hospital, London, UK

${ }^{2}$ St George's Medical School, Univeristy of London, London, UK

${ }^{3}$ Shoulder Unit, St George's Hospital London, London, UK

Corresponding author:

Robert Moverley, St Georges Hospital, Blackshaw Road, London SWI7 OQT, UK.

Email: robert.moverley@doctors.org.uk
} 
Table I. Classic anatomical descriptions of arthroscopy portals.

\begin{tabular}{|c|c|}
\hline Portal & Anatomical description \\
\hline Proximal anteromedial & $2 \mathrm{~cm}$ proximal to medial epicondyle and anterior to the intermuscular spetum 8 \\
\hline Anteromedial* & $1 \mathrm{~cm}$ to $2 \mathrm{~cm}$ anterior and $2 \mathrm{~cm}$ distal to the medial epicondyle ${ }^{\prime}$ \\
\hline Proximal anterolateral & $2 \mathrm{~cm}$ proximal and $2 \mathrm{~cm}$ anterior to the lateral epicondyle 9 \\
\hline Anterolateral* & $1 \mathrm{~cm}$ anterior and $3 \mathrm{~cm}$ distal to the lateral epicondyle ${ }^{1}$ \\
\hline Direct posterior* & $3 \mathrm{~cm}$ proximal to the olecranon tip, through the triceps tendon ${ }^{3}$ \\
\hline Proximal central posterior & $4 \mathrm{~cm}$ to $5 \mathrm{~cm}$ proximal to the olecranon tip ${ }^{4}$ \\
\hline Direct lateral portal & $\begin{array}{l}\text { Located in the middle of the triangle formed by the lateral humeral epicondyle, the } \\
\text { olecranon process and the radio-humeral joint }\end{array}$ \\
\hline Posterolateral & $3 \mathrm{~cm}$ proximal to the olecranon tip, lateral to the triceps tendon ${ }^{\prime \prime}$ \\
\hline Proximal posterolateral* & $4 \mathrm{~cm}$ to $5 \mathrm{~cm}$ proximal to the olecranon tip, lateral to the triceps tendon ${ }^{\prime \prime}$ \\
\hline
\end{tabular}

Carson $^{1}$ subjectively described the view of the elbow during arthroscopic removal of loose bodies from 12 patients. It was observed that the intra-articular surfaces of the distal humerus, trochlear ridge and coronoid were visible from the anterolateral portal. The radial head was noted to be difficult to view from the anterolateral portal. From the anteromedial portal, the capitellum and radial head could be visualized. There was no description of the trochlea notch. ${ }^{1}$

The posterior compartment has also been investigated. Adolfsson et al. ${ }^{6}$ stated that there was a limited view of the tip of the olecranon and parts of the distal humerus from the posterior portal; however, there was greater success when viewing through the direct lateral portal and using the posterior portal as a working portal. $^{6}$

Davis et al. ${ }^{12}$ attempted to quantify the amount of capitellum visible in cadaveric specimens using a standard six portal technique. By marking the FOV with a diathermy, they determined that $78 \%$ of the capitellum was accessible for instrumentation with these portals. No attempt was made to quantify the view for other anatomic regions.

The objective of the present study was to quantify the FOV available to the operating surgeon from standard arthroscopy portals using a $30^{\circ}$ arthroscope. We hypothesized that the entirety of the articular surface would be viewable using standard portals.

\section{Materials and methods}

Eleven cadaveric elbows were examined of which six were male. The cadavers were preserved using a softfix technique with a mixture of phenol and glycerol, which allows similar movements and tissue consistency to an in vivo condition. ${ }^{13}$ All cadaveric specimens were obtained and utilized in accordance with the Human Tissue Act (2004).

Whole human cadavers were positioned prone with the shoulder abducted to $90^{\circ}$ and the elbow off the edge of the examination table. This enabled the elbow to be flexed and extended, fully replicating a standard prone set-up in the operating theatre. ${ }^{4}$ A fellowship trained experienced elbow arthroscopic surgeon undertook all of the procedures.

An anterolateral portal was created as described previously ${ }^{1,3}$ (Table 1) and a standard $30^{\circ}$ 4-mm arthroscope was introduced. An anteromedial (Table 1) portal was created using a spinal needle to identify the optimum position. A $4.5-\mathrm{mm}$ full radius shaver was introduced from the anteromedial portal and used to debride any soft tissues to provide the optimum view. Throughout the procedure, the elbow was flexed and extended and the forearm pronated and supinated throughout a full range of motion to optimize the view of the articular surface from each portal.

An 18-guage spinal needle was introduced into the joint percutaneously from multiple positions to reach the limits of the FOV; this area was then marked with dye. The needle was introduced into the elbow from any position that would allow access to the visualized articular cartilage and was not confined to the use of portals. The chosen dyes had to be distinguishable from each other, remain fast and be able to stain the articular surface. The needle was inserted into the margin of the articular cartilage and Picrosius red stain was injected. Using a switching stick, the arthroscope was introduced via the anteromedial portal. After debridement of 
capsule from the anterolateral portal to improve the view the process of marking the viewable area was repeated using Nile blue stain.

A direct posterior portal was created off the tip of the olecranon and a proximal posterolateral portal was established (Table 1). With the arthroscope in the direct posterior portal, the shaver was used to expose the olecranon fossa and a spinal needle used in the same manner as for the anterior compartment to inject India ink black stain. The instruments were reversed to complete the procedure.

Once the arthroscopic procedure was completed, the soft tissues were dissected from the articular surfaces, which were then photographed using an 8-megapixel camera (Sony, Tokyo, Japan). Images were analyzed using the polygon feature of ImageJ, version $1.47 \mathrm{i}$ (National Institutes of Health, Bethesda, MD, USA), which allows for irregularly shaped selections defined by a series of line segments. The images obtained which were calibrated for accuracy.

For the purpose of the present study, the articular surface of the humerus was divided into arbitrary anterior and posterior compartments. The anterior humeral articular surface was defined as that area of the humerus lying anterior to the trans-epicondylar axis, which itself is defined as a line between the most prominent points on the epicondyles. ${ }^{14}$ The posterior humeral articular surface was defined as that area of the humerus lying behind this line. The trochlear notch of the ulnar was analyzed separately. Finally, the radial head was considered as a separate area and marked using a full range of pronation and supination.

The FOV from each portal was calculated by tracing the boundary of the cartilage demarcated with the corresponding dye, using the polygon feature in the Image J software, as shown in Figure 1.

The FOV seen from each portal was calculated for the anterior and posterior articular surfaces of the humerus, the radial head and the trochlear notch. Additionally, the total area of the anterior and posterior articular surfaces of the humerus, the radial head and the trochlea were measured, allowing the percentage of viewable area (FOV percentage) to be calculated. The FOV percentage area was calculated to provide a comparison for the FOV in each cadaver. Total FOV percentage areas were compared using a Student's $t$-test (JMP, version 10). $p<0.05$ was considered statistically significant.

\section{Results}

Data from 11 elbows were analyzed to quantify the FOV obtainable from the standard portals. For the measurement of the total area seen of the radial head and trochlear notch, one cadaver was excluded as a result of severe osteoarthritis.

The anterior articular surface of the humerus was viewed from the anteromedial and anterolateral portals. The mean (SD) FOV percentage area seen from the anteromedial portal and anterolateral portal was

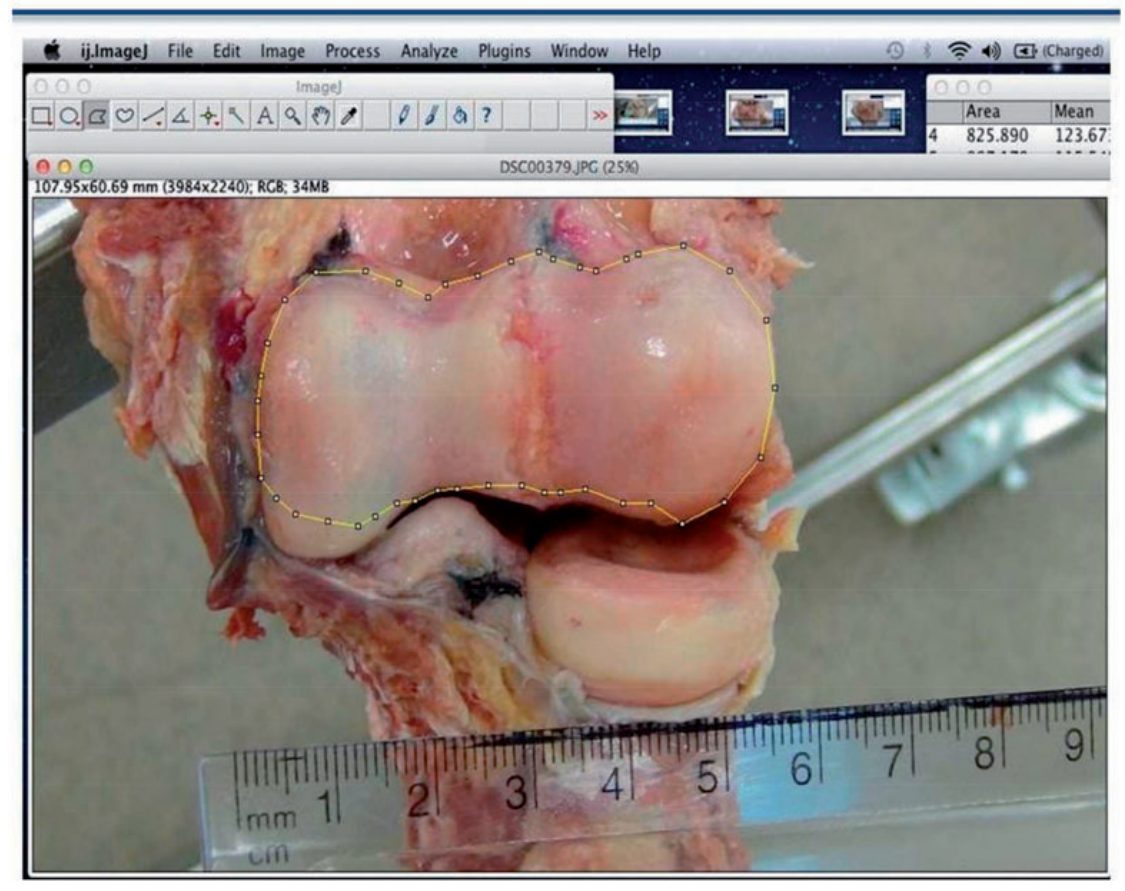

Figure I. Illustration of the polygon feature of ImageJ used to measure the areas marked. 


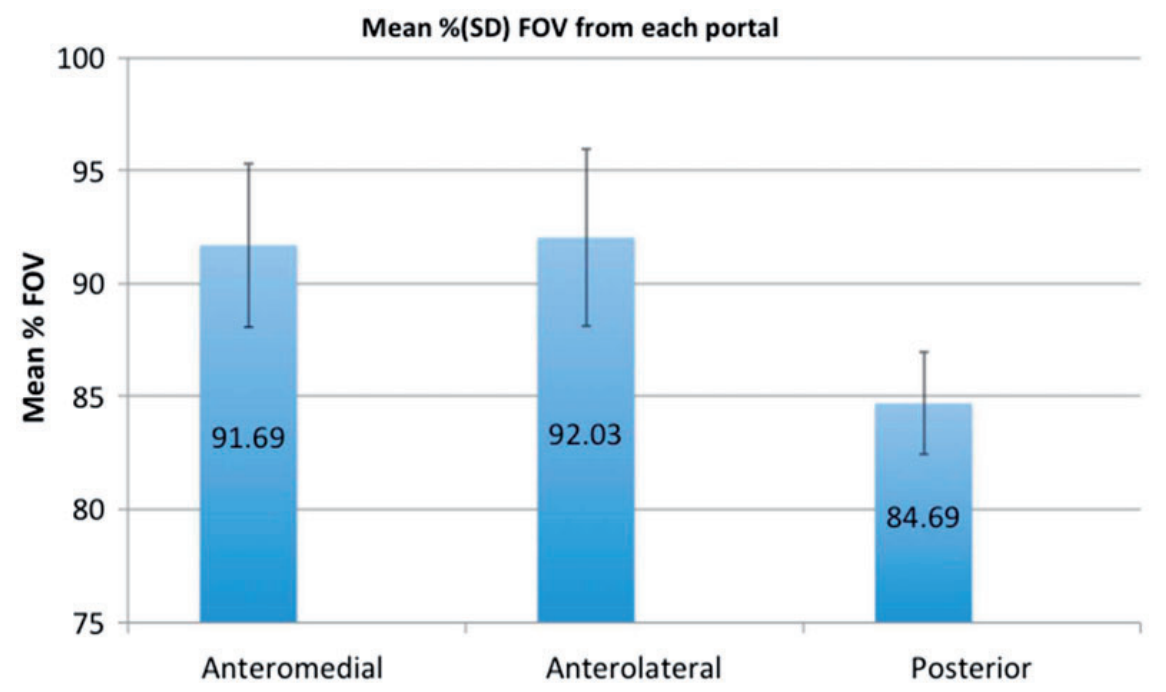

Figure 2. Mean percentage of articular surface visible from each portal.

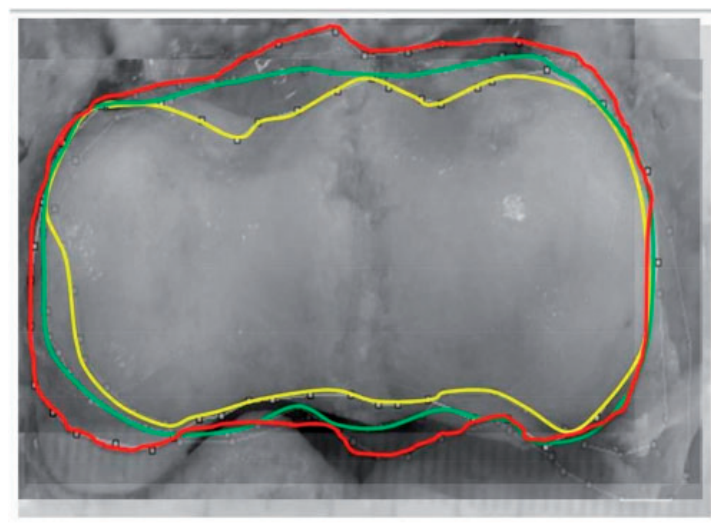

Figure 3. A composite image to show the maximum (red), minimum (yellow) and mean (green) total field of view (FOV) percentage area seen of the anterior aspect of the elbow joint from the anteromedial and anterolateral portals.

$91.69 \%(3.63 \%)$ and $92.03 \%(3.93 \%)$, respectively. There was no statistical difference seen in the mean FOV percentage area seen from the anteromedial and anterolateral portal $(p=0.99)$. The posterior humerus articular surface was viewed through direct posterior and proximal posterolateral portals. The mean (SD) FOV percentage area seen from the posterior portals was $84.69 \%(2.28 \%)$ (Figure 2).

The mean composite images demonstrate that the majority of the anterior aspect of the elbow joint could be seen from both the anteromedial and the anterolateral portals (Figure 3)

The mean posterior composite images showed that the region covered by the tip of the olecranon could not be seen (Figure 4).

The mean (SD) FOV percentage area seen from all three portals of the radial head was $16.05 \%(2.66 \%)$.

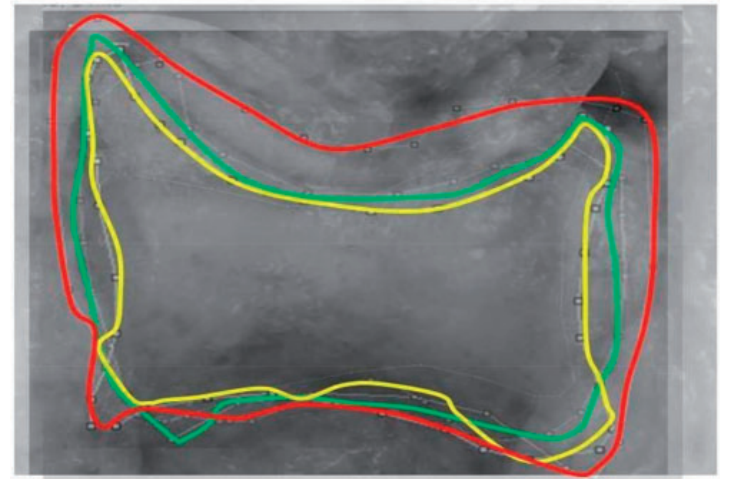

Figure 4. A composite image to show the maximum (red), minimum (yellow) and mean (green) total field of view (FOV) percentage area seen of the posterior aspect of the elbow joint from the posterior portals.

The mean (SD) FOV percentage area seen from all three portals of the trochlear was $4.14 \%(1.76 \%)$ (Figure 5).

Composite images of the radial head and trochlear notch were compiled. The radial head composite images showed that only the lateral aspect of the radial head could be seen (Figure 6).

The trochlear notch composite images showed only the anterior ridge of the trochlear notch could be seen (Figure 7).

\section{Discussion}

The indications for elbow arthroscopy are expanding. There is a body of evidence regarding optimum portal placement but almost all of this is in reference to avoiding damage to the neurovascular structures rather than 


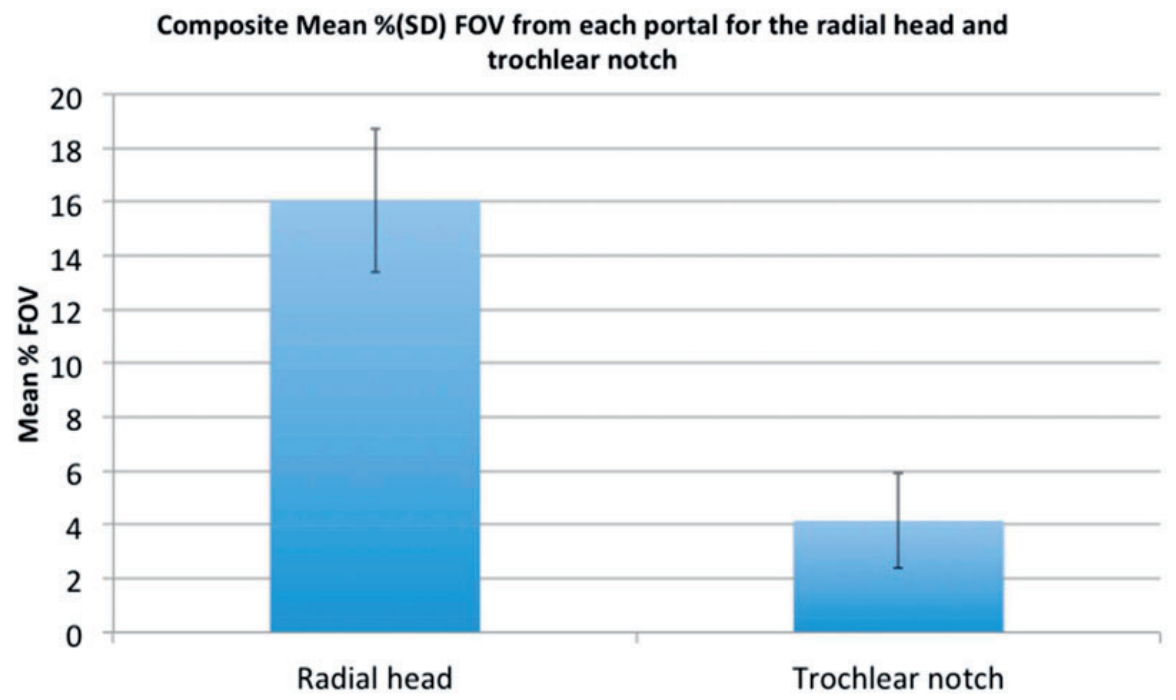

Figure 5. Mean composite percentage of articular surface visible from all three portals for the radial head and trochlear notch.

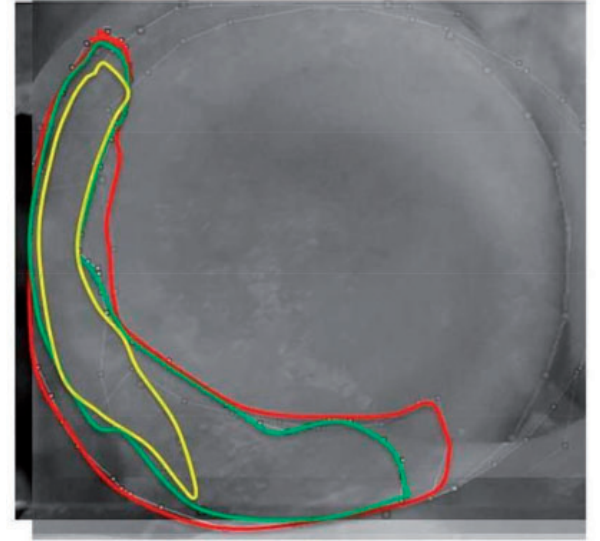

Figure 6. A composite image to show the maximum (red), minimum (yellow) and mean (green) total field of view (FOV) percentage area seen of the radial head from the medial and lateral portals.

optimizing the field of view. A few studies have reported the limitations but so far no attempt has been made to quantify this.

The results of the present study demonstrate the mean FOV percentage area seen of the anterior articular surface of the distal humerus to be $91.69 \%$ from the anteromedial portal and $92.03 \%$ from the anterolateral portal. The limitation in the FOV from the anteromedial portal was noted to be the lateral aspect of the capitellum and close to the articulating surface of the trochlear notch. The limitations in the FOV from the anterolateral portal were noted to be mainly the ridge of the trochlear notch and the radiohumeral articulation. These limitations can be seen visually in the composite anterior view (Figures 6 and 7) whereby

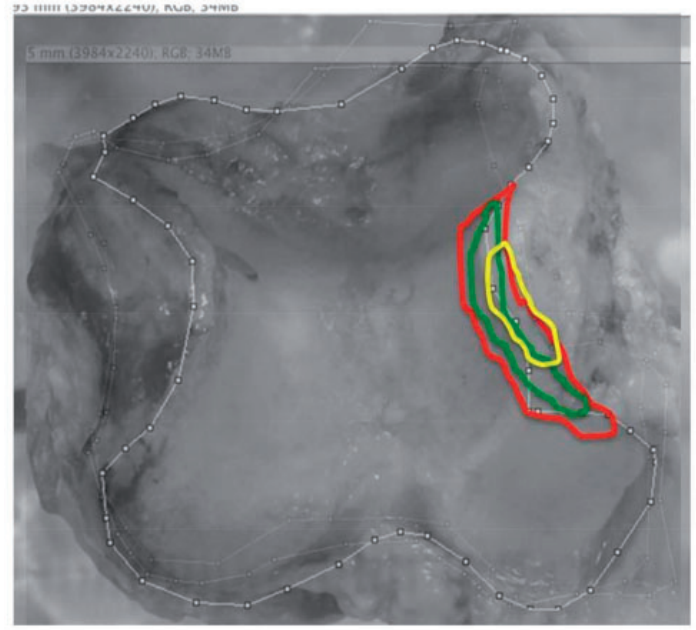

Figure 7. A composite image to show the maximum (red), minimum (yellow) and mean (green) total field of view (FOV) percentage area seen of the trochlear notch from the medial and lateral portals.

only the lateral aspect of the radial head and anterior ridge of the trochlear notch can be seen. On this basis, a surgeon should be cautious in ruling out or expecting to treat pathology in the radial head and trochlear using only these portals and may wish to consider the use of accessory portals.

An evaluation of the arthroscopic technique in the knee showed a blind spot in the visual field of the medial meniscus and concluded that it was a result of the degree of curvature. ${ }^{15}$ This concept can be applied to the anatomy of the capitellum; thus, the limitation in FOV that we found in the capitellum could be a result of its curvature. The findings of the present study are 
consistent with other studies that have found less than satisfactory results for procedures regarding the capitellum. ${ }^{1,6}$ Baumgartner suggested that osteochondrotic lesions of the capitellum could be missed if viewed entirely from the anterior portals and described using a direct lateral portal and a smaller arthroscope to overcome this. ${ }^{16}$ Davis et al. ${ }^{12}$ were able to view $78 \%$ of the capitellum using a dual lateral portal technique.

The results of this investigation demonstrate that both the anterolateral and anteromedial portals afford an excellent view of the anterior articular surface. It is noted that the limit in the FOV from the anteromedial portal was seen to be the lateral aspect of the capitellum, whereas the capitellum was within the central FOV from the anterolateral portal.

Although there was no statistically significant difference between the mean total percentage area seen from both anterior portals, the anatomical regions seen from each portal are different. Therefore, by switching portals, a surgeon can view and instrument an even larger proportion for the anterior compartment than reported in the present study.

The present study demonstrates that $84.69 \%$ of the posterior humerus articular surface could be seen from the posterior portal. It was noted, in the composite image for the posterior portal (Figure 4), that the region covered by the tip of the olecranon was the most difficult to visualize. This is in keeping with previous cadaveric study by Adolfsson et al. ${ }^{6}$ that demonstrated similar limitations in the view of the tip of the olecranon fossa, especially when viewed from the posterior portal. In a review of the elbow arthroscopic technique, Bennet ${ }^{5}$ concluded that the posterior portal showed the entire posterior aspect of the joint but this was a purely descriptive, with no attempt to quantify the FOV.

Steinmann ${ }^{2}$ concluded that the viewing potential from the posterior portal is slightly limited due to the posterior fat pad obscuring some of the FOV. This is a consistent finding and the effect was minimized in the present study by the use of a shaver to remove the fat pad and any other soft tissue, which may have been obscuring the view. It is questionable whether this aggressive debridement would be suitable for each patient in the clinical setting.

The FOV of the trochlear notch from the anterior portals was limited, with an average FOV percentage area of $4.14 \%$, and this view was confined exclusively to the trochlear ridge as seen in the composite view (Figure 7). This finding is consistent with previous studies. Andrews and Carson ${ }^{1}$ state that the trochlear ridge could be seen but did not describe whether the entire trochlea notch could be seen ${ }^{1}$. Adolfsson et al. ${ }^{6}$ noted the medial aspects of the ulnohumeral joint were difficult to visualize. Timmerman and Andrews ${ }^{17}$ concluded that the trochlear groove was very difficult to reach via arthroscopy or open surgery. The lack of visual field would be most likely be attributable to the congruency of the ulnohumeral joint, thus providing insufficient area for the arthroscope and needle to demarcate a significant FOV. Miller et al. ${ }^{18}$ suggest the radial head and trochlea are visible using the direct lateral and accessory lateral portals and recommend a small wrist scope $(2.7 \mathrm{~mm})$ to do this.

The present study suggests that only $16.05 \%$ of the radial head could be seen from all three portal sites and that this was predominantly confined to the lateral rim despite a full range of supination and pronation being employed. These findings are contradictory to those of Adolfsson et al. ${ }^{6}$ who concluded that approximately $100^{\circ}$ of the rim of the radial head could be seen from the lateral portal and the concave radial head could not be seen. Andrews and Carson ${ }^{1}$ stated the FOV for the radial head was limited from the anterolateral portal but that this could be improved using the anteromedial portal. The improved visualization of the radial head from the anteromedial portal was not replicated in the present study. However, a previously mentioned limitation of the present study is that the proportion of the radial head that could be visualized was far more than that which could be marked with a straight needle. It was considered that the results significantly underestimate the true visualization area but agree with the findings of Adolfsson et al. ${ }^{6}$ with respect to the area being predominantly confined to the rim.

There are limitations to the present study; first, the use of cadavers may not be a true representation of the FOV in younger patients without arthritis. It is also important to differentiate between what is viewable and what is accessible to a straight spinal needle. Allowing the surgeon to access the joint via any point on the skin minimized this effect, although the concave surface of the radial head remained inaccessible. Therefore, although arthroscopy may allow the surgeon to view more area than is reported in the present study, the ability to access all that is seen with instrumentation is more limited and may be a more accurate reflection of the results of the current investigation. Furthermore, pathology in the elbow is often outside the margin of articular cartilage on the anterior aspect of the humerus and tip of the coronoid, which has not been assessed.

The present study has utilized a technique for measuring the FOV afforded by previously described 'standard' arthroscopy portals using a $30^{\circ}$ arthroscope. This study has not, however, validated the technique or attempted to assess inter-oberserver and intra-observer reliability. 
We acknowledge that other portals are available, and that the FOV may be increased by viewing from additional portals or by using either a $70^{\circ}$ or $2.7-\mathrm{mm}$ arthroscope. The present study highlights the limitations of the standard portals, and surgeons should plan optimum portal position to maximize view and allow instrumentation of suspected pathology. All portals will essentially be a compromise and, in providing an excellent view of one aspect of the joint, it may be more difficult to assess pathology in another region.

Future work is needed to quantify whether the FOV can be significantly improved using accessory portals and nonstandard arthroscopic instruments.

\section{Conclusions}

The present investigation has confirmed that there are limitations of the FOV in elbow arthroscopy using standard portals and a $30^{\circ}$ arthroscope and is the first study to attempt to quantify the deficit. If one accepts that the results obtained for the radial head are unlikely to be accurate because of the associated difficulty in instrumentation, the most significant limitations in the FOV were observed in the trochlear notch. We have demonstrated that the majority of the capitellum could be seen from the anteromedial and anterolateral portals, with the exception of its far lateral aspect. The present study highlights the limitations of elbow arthroscopy and surgeons should be cautious about diagnosing and treating trochlear pathology with this technique. Further work is required to quantify the true visible area of the radial head.

\section{Declaration of Conflicting Interests}

The author(s) declared no potential conflicts of interest with respect to the research, authorship, and/or publication of this article.

\section{Funding}

The author(s) received no financial support for the research, authorship, and/or publication of this article.

\section{References}

1. Andrews JR and Carson WG. Arthroscopy of the elbow. Arthroscopy 1985; 1: 97-107.

2. Steinmann SP. Elbow arthroscopy: where are we now? Arthroscopy 2007; 23: 1231-1236.
3. Poehling GG, Whipple TL, Sisco L and Goldman B. Elbow arthroscopy: a new technique. Arthroscopy 1989; 5: 222-224.

4. Plancher KD and Bishai SK. Basics of elbow arthroscopy: setup, portals, and technique. Tech Orthop 2006; 21: 239-249.

5. Bennett JM. Elbow arthroscopy: the basics. J Hand Surg 2013; 38: 164-167.

6. Adolfsson L. Arthroscopy of the elbow joint: A cadaveric study of portal placement. J Shoulder Elbow Surg 1994; 3: 53-61.

7. Unlu MC, Kesmezacar H, Akgun I, Ogut T and Uzun I. Anatomic relationship between elbow arthroscopy portals and neurovascular structures in different elbow and forearm positions. J Shoulder Elbow Surg 2006; 15: 457-462.

8. Lindenfeld TN. Medial approach in elbow arthroscopy. Am Journal Sports Med 1990; 18: 413-417.

9. Field LD, Altchek DW, Warren RF, O'Brien SJ, Skyhar MJ and Wickiewicz TL. Arthroscopic anatomy of the lateral elbow: a comparison of three portals. Arthroscopy 1994; 10: 602-607.

10. Morrey BF. The elbow and its disorders. Philadelphia, PA: WB Saunders, 1985.

11. Stothers K, Day B and Regan WR. Arthroscopy of the elbow: anatomy, portal sites, and a description of the proximal lateral portal. Arthroscopy 1995; 11: 449-457.

12. Davis JT, Idjadi JA, Siskosky MJ and ElAttrache NS. Dual direct lateral portals for treatment of osteochondritis dissecans of the capitellum: an anatomic study. Arthroscopy 2007; 23: 723-728.

13. Thiel W. [The preservation of the whole corpse with natural color]. Ann Anat 1992; 174: 185-195.

14. Sabo MT, Athwal GS and King GJ. Landmarks for rotational alignment of the humeral component during elbow arthroplasty. J Bone Joint Surg Am 2012; 94: 1794-1800.

15. Morin WD and Steadman JR. Arthroscopic assessment of the posterior compartments of the knee via the intercondylar notch: the arthroscopist's field of view. Arthroscopy 1993; 9: 284-290.

16. Baumgarten TE, Andrews JR and Satterwhite YE. The arthroscopic classification and treatment of osteochondritis dissecans of the capitellum. Am J Sports Med 1998; 26: 520-523.

17. Timmerman LA and Andrews JR. Arthroscopic treatment of posttraumatic elbow pain and stiffness. Am J Sports Med 1994; 22: 230-235.

18. Miller D, Gregory JJ and Hay SM. Arthroscopy of the elbow. Curr Orthop 2008; 22: 104-110. 